\title{
Avaliação e controle de contatos faltosos de doentes com Hanseníase
}

\author{
Evaluation and control of missing contacts of Leprosy patients \\ Evaluación y control de contactos faltantes de enfermos con Lepra
}

\section{Carmen Silvia de Campos Almeida Vieira', Marina Teixeira Soares', Cláudia Tatiana Siqueira Xavier Ribeiro', Letícia Faroni Guimarães da Silva'}

'Universidade de Taubaté. Departamento de Enfermagem. Taubaté, SP

Submissão: 29/08/2008

Aprovação: 10/10/2008

\section{RESUMO}

Estudo descritivo, com variáveis Qualitativas, realizado no Ambulatório Regional de Especialidades de Taubaté, com objetivo de resgatar e avaliar contatos faltosos de doentes de hanseníase. Analisou-se 36 prontuários de casos novos, no período de janeiro de 2003 a julho de 2004. Identificou-se 92 contatos, sendo 64, I \% faltosos, tendo $25 \%$ entre 20 a 29 anos e $58,5 \%$ do sexo feminino. Destes, $61 \%$ foram submetidos à consulta de enfermagem no domicilio. Relataram não adesão ao controle por esQuecimento $(66,6 \%)$, e falta de tempo ( 1 1, 1\%); 4 contatos tinham sintomas de hanseníase, e I foi confirmado (forma transmissível - Dimorfa). Sem atingir 100\% da cobertura de busca ativa, sugerimos, para assegurar a meta de eliminação da doença, parcerias e descentralização das ações de controle.

Descritores: Medidas em epidemiologia; Hanseníase/transmissão; Período de transmissibilidade; Enfermagem.

\section{ABSTRACT}

Descriptive study, with Qualitative variables, carried on in the Ambulatory of Specialties Region of Taubaté, in order to evaluate the missing contacts of leprosy patients. Thirty-six records of new cases were analyzed, from January 2003 to July 2004. Ninety-two contacts were identified, being $64.1 \%$ missing ones, having $25 \%$ from 20 to 29 years and $58.5 \%$ female. Of these, $61 \%$ were submitted to consultation of home nursing. Reported non-membership to the control for forgetfulness (66.6\%), and lack of time (1 1.1\%), 4 contacts had symptoms of leprosy, and I was confirmed (transmissible form - Dimorfa). Without achieving 100\% coverage active search, we suggest, to ensure the goal of eliminating of the disease, partnerships and decentralization of the control activities.

Descriptors: Epidemiologic measurements; Leprosy/transmission; Communicable period; Nursing.

\section{RESUMEN}

Estudio descriptivo, con cualitativas variable, realizado en el Ambulatorio de Especialidades Regional de Taubaté, a fin de canjear y evaluar las deficiencias de enfermos de lepra. Se analizaron los registros de 36 nuevos casos, entre enero de 2003 y julio de 2004 . Se identificaron 92 contactos, sendo 64, I \% deficiencias, tendo 25\% de 20 a 29 años y 58,5\% mujeres. De estos, $61 \%$ fueron sometidos a consulta en la casa de los enfermos. Informó de la no pertenencia al control en el olvido $(66,6 \%)$, y la falta de tiempo ( 11 , 1\%), 4 contactos había síntomas de la lepra, y se confirmó I (formulario transmisibles - Dimorfa). Sin lograr una cobertura del 100\% del activo de bús@ueda, le sugerimos Que, para garantizar el objetivo de eliminar la enfermedad, las asociaciones y la descentralización de las actividades de control. Descriptores: Mediciones epidemiológicas; Lepra/transmisión; Periodo de transmisión; Enfermería. 


\section{INTRODUÇÃO}

A hanseníase é uma doença causada pelo Mycobacterium leprae, ou bacilo de Hansen, um parasita intracelular obrigatório, com afinidade por células cutâneas e nervos periféricos, Que se instala no organismo da pessoa infectada, podendo se multiplicar. A transmissão se faz de forma direta, por via respiratória, sendo necessário ter predisposição para adouirir a doença e ter contato íntimo e prolongado com o doente sem tratamento ${ }^{(1)}$. $O$ bacilo tem a capacidade de infectar um grande número de pessoas, mas, somente $10 \%$ das Que vivem em situações de alta prevalência adoecem. $\mathrm{O}$ aparecimento da doença na pessoa infectada pelo bacilo, e suas diferentes manifestações clínicas dependem dentre outros fatores, da relação parasita/ hospedeiro e pode ocorrer após um longo período de incubação, de 2 a 7 anos ${ }^{(2)}$

Na maioria das regiões do mundo a incidência da doença é maior nos homens do Que nas mulheres. Existem outros fatores Que favorecem a endemicidade, como as condições socioeconômicas desfavoráveis, condições precárias de vida e de saúde, e o elevado número de pessoas convivendo em um mesmo ambiente influem no risco de adoecer ${ }^{(2)}$.

A doença, inicialmente, manifesta-se por meio de lesões de pele: manchas esbranquiçadas ou avermelhadas Que apresentam perda de sensibilidade. Estas lesões ocorrem em Qualquer região do corpo, mas, com maior freeüência, na face, orelhas, nádegas, braços, pernas, costas e mucosa nasal. Se não tratada, manifestam-se as lesões nos nervos, principalmente nos troncos periféricos. Podem aparecer nervos espessados e doloridos, diminuição de sensibilidade nas áreas inervadas, resultando em comprometimento sensitivo, motor e autonômico (alterações de glândulas sudoríparas e sebáceas), responsáveis pelas incapacidades e deformidades, características da hanseníase ${ }^{(2)}$.

Os doentes de hanseníase são classificados como Paucibacilares e Multibacilares. Os paucibacilares são os doentes nas formas clínicas indeterminadas e tuberculóides, abrigam um pequeno número de bacilos, insuficientes para infectar outras pessoas, têm baciloscopia negativa, e apresentam menos de 5 lesões de pele e ou apenas um tronco nervoso acometido. Já os Multibacilares, forma contagiosa da hanseníase abrigam um grande número de bacilos, têm baciloscopia positiva, são classificados como virchovianos e dimorfos; apresentam mais de 5 lesões de pele e ou mais de um tronco nervoso acometido, sendo os Que não estão em tratamento considerados fontes de transmissão e infecção( ${ }^{(3)}$. O tratamento é indispensável ao paciente para Que possa curar-se, fechando a fonte de infecção e interrompendo a transmissão da doença, sendo então estratégico no controle da endemia e para a eliminação da hanseníase ${ }^{(2)}$. O acompanhamento é ambulatorial na rede de serviço público, onde o doente deve comparecer para consulta médica e de enfermagem, receber a dose supervisionada do tratamento polieuimioterápico (PQT), e avaliação de prevenção de incapacidades (PI). A duração do tratamento varia de acordo com a classificação da doença; sendo paucibacilar, sua duração é de 6 em até 9 meses, e multibacilar, de 12 em até 18 meses, ou 24 meses em casos mais complexos ${ }^{(4)}$.

A hanseníase hoje representa um grave problema de Saúde Pública no Brasil, além dos agravantes inerentes a QualQuer doença de origem sócio-econômica; ressalta-se, assim, a repercussão psicológica gerada pelas incapacidades físicas, advindas da doença, Quando não devidamente tratada. Estas incapacidades constituem, na realidade, a grande causa do estigma e isolamento do paciente na sociedade ${ }^{(5)}$. Para evitá-las, as ações da PI englobam: educação em saúde; diagnóstico precoce da doença; tratamento regular da PQT, e controle de contatos intradomiciliares, incluindo a aplicação de vacina BCGID; detecção precoce e tratamento adeQuado das reações e neurites; apoio à manutenção da condição emocional e integração social (família, estudo, trabalho, grupos sociais) e orientação Quanto a realização de autocuidado ${ }^{(6)}$.

Todos portadores de hanseníase foram primeiramente comunicantes, portanto são considerados de importância epidemiológica significativa em termos de endemia e passam a se tornar um grupo de risco vulnerável do ponto de vista da cadeia do processo infeccioso. Uma das propostas de atingir as metas de eliminação é de controlar os contatos intradomiciliares, Que são as pessoas Que residem na mesma casa ou Que tenham um convívio contínuo e prolongado com o doente ${ }^{(7)}$. Os dados epidemiológicos da cobertura de controle desses contatos no Brasil em 2007 atingiram apenas $47 \%{ }^{(8)}$, e como esta população está mais susceptível a adoecer, este foi o motivo Que estimulou o desenvolvimento deste estudo.

A pesquisa teve como objetivo geral, realizar a busca ativa dos contatos intradomiciliares faltosos no controle de hanseníase em um Ambulatório Regional de Especialidades do Vale do Paraíba, e como objetivos específicos, caracterizar o perfil dos contatos intradomiciliares e os motivos da não adesão ao controle; realizar consulta de enfermagem dos contatos faltosos e identificar e encaminhar os casos suspeitos para avaliação e conduta.

\section{MÉTODO}

Tratou-se de um estudo descritivo, exploratório, com variáveis Qualitativas, para aprofundar os conhecimentos sobre os motivos da não assiduidade no controle dos contatos intradomiciliares. Desenvolveu-se em duas etapas: a primeira foi embasada na pesquisa documental, e a segunda foi por meio de visita domiciliar e consulta de enfermagem, para identificar os sinais suspeitos de hanseníase e encaminhamento para elucidação do diagnóstico.

O estudo realizou-se no Ambulatório Regional de Especialidades (ARE) do município de Taubaté, localizado no Vale do Paraíba, interior do Estado de São Paulo. O município conta com uma população estimada de 265.514 habitantes, Quase a sua totalidade na zona urbana $^{(9)}$. O ARE Taubaté tem por finalidade prestar assistência ambulatorial à comunidade em nível secundário e responder como Centro Formador e Capacitador de Recursos Humanos nos Programas de Saúde da Secretaria de Estado da Saúde, Constitui-se Unidade de Referência, na DRS-XVII, Que abrange 33 municípios da região do Vale do Paraíba ${ }^{(10)}$. Em relação à hanseníase o ARE, tornou-se uma unidade de referência para atender os casos mais complexos de diagnóstico, casos de reação hansênica, ações de prevenção de incapacidade (PI) de média complexidade, curativos, e encaminhamentos para adaptação e confecção de calçados.

Para a seleção da amostra estudaram-se prontuários, fichas epidemiológicas e de acompanhamento, de 100\% de casos novos de pacientes inscritos no Programa de Controle de Hanseníase, no período de janeiro de 2003 a julho de 2004. Deste levantamento, identificou-se 36 doentes, sendo 18 paucibacilares e 18 multibacilares, com contatos intradomiciliares, totalizando 92 
contatos. Destes contatos, 59 eram faltosos, dos Quais 36 aceitaram participar da peseuisa, sendo submetidos à visita domiciliar e consulta de enfermagem.

Estudou-se a população selecionada de acordo com os indicadores epidemiológicos do foco (forma clínica, baciloscopia, data do início do tratamento, esquema terapêutico), e do contato (número de contatos registrados, controle do BCG-ID, e a assiduidade ao exame dermatoneurológico).

Inicialmente para cumprir as normas estabelecidas pela resolução do Conselho Nacional de Saúde 196/96 ${ }^{(11)}$, o projeto foi submetido à apreciação do Comitê de Ética em Pesquisa da Universidade de Taubaté (Protocolo CEP/UNITAU n ${ }^{\circ}$ 004/07), e aprovado.

Foi elaborado e aplicado um formulário, Que contemplou os dados de identificação dos contatos intradomiciliares selecionados, sendo, posteriormente, realizado seu exame dermatoneurológico (céfalocaudal). Para identificação dos participantes do estudo (foco e contato intradomiciliar), foram selecionadas as seguintes variáveis: idade, sexo, condições de saneamento e moradia, forma clinica, procedência, ocupação, data de inicio do tratamento do foco, resultados de baciloscopia, motivo do não comparecimento para o controle de contatos.

Para consolidação dos dados foram elaborados três instrumentos para alimentação do banco de dados (Excel 98): Ficha de Registro de dados dos Contatos Intradomiciliares, Ficha de Consolidação dos Contatos Faltosos e Formulário para Consulta de Enfermagem.

Durante a realização da consulta de enfermagem, foram primeiramente identificados todos os contatos faltosos, explicados os objetivos da pesquisa e o motivo da visita, bem como a leitura do "Termo de Consentimento Livre e Esclarecido". Após a aceitação e assinatura do termo, realizou-se trabalho educativo sobre hanseníase, por meio de um álbum seriado da Fundação Paulista contra a Hanseníase do Estado de São Paulo. Na residência do contato faltoso escolheu-se um local mais reservado para realizar a consulta, Que contemplava levantamento de dados pessoais, Queixas, exame dermatoneurológico, com pesquisa da sensibilidade por meio do estesiômetro, verificação da situação vacinal do BCG-ID, agendamento e encaminhamentos dos casos suspeitos. Foram avaliados 36 contatos intradomiciliares; para isso, foram necessárias as realizações de algumas visitas nos finais de semana, devido ao fato dos contatos estarem inseridos no mercado de trabalho sem disponibilidade de serem avaliados no horário comercial.

\section{RESULTADOS E DISCUSSÃO}

Para melhor elucidação dos resultados encontrados, optou-se por descrever sobre a caracterização dos focos e de seus contatos intradomiciliares. Quanto à patologia estudada, por se tratar de uma doença infecto contagiosa, foi valorizada também, a situação epidemiológica do foco, de seus contatos intradomiciliares, enfatizando a forma clinica, assiduidade, formas de intervenção (convocação por telefone, correio; consulta de enfermagem e visita domiciliaria), suspeição diagnóstica e encaminhamentos.

Os dados coletados foram apresentados em forma de tabelas e figuras, para melhor observação e análise.

A primeira Tabela representa os contatos intradomiciliares faltosos, conforme a forma clínica, o sexo e a faixa etária do foco.

A Tabela I demonstra Que a maioria dos contatos $(66,6 \%)$, é de focos multibacilares (forma dimorfa - 49,9\% e virchoviana - 16,7\%), seguidos das formas paucibacilares, $(33,4 \%$, sendo: forma indeterminada - 11, 1\% e tuberculóide - 22,3\%).

Um estudo realizado ${ }^{(12)}$ no Rio de Janeiro, no período de 1987 a 1991, detectou predominância das formas multibacilares dos casos primários dos contatos da coorte, chegando a 72,2\% das formas clínicas dos casos-índice, semelhantes ao encontrado nesta pesquisa. Este fato é confirmado por estudiosos ${ }^{(13)}$ Que referem: "Quanto mais íntima e duradoura for à convivência com um doente bacilífero sem tratamento, maior é a possibilidade de se contrair a doença. Por isso, em países com prevalência como a nossa, a busca de casos deve ser feita prioritariamente entre os contatos domiciliares de pacientes bacilíferos".

Quanto à faixa etária dos estudados, predominaram: de 20 a 29 (27,7\%), 10 a 19 (25\%) e 0 a $9(19,4 \%)$, ou seja os contatos estudados estão concentrados na fase produtiva da vida e na faixa etária mais jovem. Em países endêmicos, a população infantil entra precocemente em contato com doentes bacilíferos, o que colabora para detecção da doença entre crianças de três a cinco anos, sendo raramente observados casos em menores de dois anos, principalmente da forma Virchoviana. Esses dados demonstram o risco dos menores de 15 anos adoecerem de hanseníase em países endêmicos ${ }^{(14)}$; fato este semelhante ao encontrado neste estudo.

Em relação ao sexo, encontrou-se entre os contatos intradomiciliares um maior número de mulheres $(58,5 \%)$, do Que os homens $(41,6 \%)$, dados estes semelhantes ao encontrados em outro estudo $^{(12)}$,onde o percentual maior (58\%) de contatos eram do sexo feminino.

Na tabela 1, correlacionando-se sexo, faixa etária e forma clinica, identificam-se maior percentual de casos da forma dimorfa (49,9\%), do sexo feminino $(27,8 \%)$ e das faixas etárias 0 a 9, e 20 a 29 anos, com percentual de $8,3 \%$, respectivamente.

Tabela 1. Distribuição dos contatos faltosos de hanseníase, segundo a forma clínica, sexo e faixa etária do foco.

\begin{tabular}{|c|c|c|c|c|c|c|c|c|c|}
\hline \multirow{3}{*}{ Faixa Etária } & \multicolumn{8}{|c|}{ Forma Clínica } & \multirow{3}{*}{ Total } \\
\hline & \multicolumn{2}{|c|}{ I } & \multicolumn{2}{|c|}{$T$} & \multicolumn{2}{|c|}{ D } & \multicolumn{2}{|c|}{ V } & \\
\hline & $\mathrm{F}$ & $M$ & $\mathrm{~F}$ & $\mathrm{M}$ & $F$ & $M$ & $F$ & $\mathbf{M}$ & \\
\hline $0-9$ & - & - & - & 1 & 3 & - & 1 & 3 & 8 \\
\hline $10-19$ & 1 & - & 2 & 1 & 1 & 2 & 1 & - & 8 \\
\hline $20-29$ & 2 & - & - & 1 & 3 & 3 & - & - & 9 \\
\hline $30-39$ & - & - & 1 & - & 1 & 1 & 1 & - & 4 \\
\hline$>60$ & - & - & - & - & 1 & - & - & - & 1 \\
\hline Total & 4 & 0 & 4 & 4 & 10 & 8 & 3 & 3 & 36 \\
\hline
\end{tabular}


Em relação à ocupação dos contatos, verificou-se um percentual significativo de estudantes (33,3\%), Que é o esperado Quando comparamos com os dados da Tabela I, Que demonstra um grande percentual de contatos jovens $(69,4 \%)$. Observou-se também um número expressivo de contatos trabalhando nas seguintes atividades: metalúrgica e do lar $(8,3 \%)$ respectivamente. Portanto, no estudo, verificou-se um percentual significante de contatos Que não estão inseridos no mercado de trabalho (16,7\%), o Que vem de encontro com a atual situação de desemprego no Brasil, como o apresentado pela Pesquisa de Emprego e Desemprego (PED), Fundação Seade/ Dieese, desenvolvida pelo.Instituto de Geografia e Estatística (IBGE), em março de 2007, Que registrou um aumento do desemprego, com taxa de desocupação passando de 15,9\%, em fevereiro, para $16,6 \%$, em março ${ }^{(9)}$.

O estudo Identificou Que $100 \%$ dos contatos residiam na zona urbana; $80,6 \%$ possuíam casa própria, com abastecimento de água, destino final de lixo, e esgoto, porém uma parcela dos contatos $(19,4 \%)$ não possuía abastecimento de água encanada, utilizando água de poço; fossa para eliminação de dejetos, e não tinha coleta de lixo em suas residências, desenvolvendo a prática da Queima dos mesmos.

Estudiosos referem ${ }^{(15)}$ a importância de valorizar Que as doenças endêmicas como a hanseníase e a tuberculose sofrem influências, de um lado pelo nível de desenvolvimento sócio econômico e por outro lado, pelas condições de vida da população. Relatos sobre o tema já expressavam Que muitas variáveis são indicativas de pobreza; de más condições de moradia; de falta de acesso aos serviços públicos, como saneamento, água encanada; de falta de higiene, e estes fatores estiveram associados significativamente à hanseníase ${ }^{(16)}$ Estes fatores vêm corroborar com o presente estudo, Que apresentou dados semelhantes.

Na Figura I será explicitado o total de contatos em registro, a incidência de faltosos e o percentual dos que foram submetidos à visita domiciliar e consulta de enfermagem, no período estudado.

A Figura I demonstra Que 92 (100\%) eram o total de contatos registrados no Programa de Controle da Hanseníase, destes, 64, $1 \%$ eram faltosos. Dos faltosos, somente $61 \%$, foram avaliados por meio da visita domiciliar e consulta de enfermagem, sendo que os demais faltosos não foram avaliados, por motivos Que serão discutidos na Tabela 2. O estudo demonstra a dificuldade de se resgatar os contatos intradomiciliares faltosos, mesmo Quando se faz a busca ativa em suas residências.

Alguns autores discutem propostas de melhoria deste controle Que podem ser explicitados nos relatos a seguir: "Na rede de serviços de saúde Que desenvolvem as ações de controle da hanseníase, são apontadas deficiências Que comprometem tanto a assistência aos doentes Quanto aos comunicantes, alimentando os Que os hansenólogos denominam de "iceberg epidemiológico" da endemia hansênica"(17); "... a melhor estratégia é incentivar o exame dos contatos pela educação dos pacientes, da família e da comunidade ${ }^{(18)}$.

Apesar dos esforços no sentido de resgatar a população do estudo, por meio da busca ativa, em um curto espaço de tempo, verificaramse dificuldades para atingir a meta de $100 \%$. Isto demonstra o Quanto é complexo desenvolver as ações de controle de contatos, principalmente Quando as consultas dos mesmos devem ser mantidas por um período maior do Que o estabelecido para o controle dos doentes. Os contatos de doentes paucibacilares, devem ser acompanhados por dois anos a partir da data do inicio do tratamento do doente, e os contatos de doentes multibacilares acompanhados por $5 \operatorname{anos}^{(2)}$, fato este corroborado por pesquisador ${ }^{(18)}$, Que reforça a importância da educação continuada com os contatos.

A Tabela 2 demonstra os motivos da não avaliação dos contatos na consulta de enfermagem. Dos 23 contatos intradomiciliares, 43,5\% não foram avaliados por não estarem em sua residência no momento da visita dos pesquisadores, e mesmo após um novo agendamento não compareciam no ARE na data programada. Os

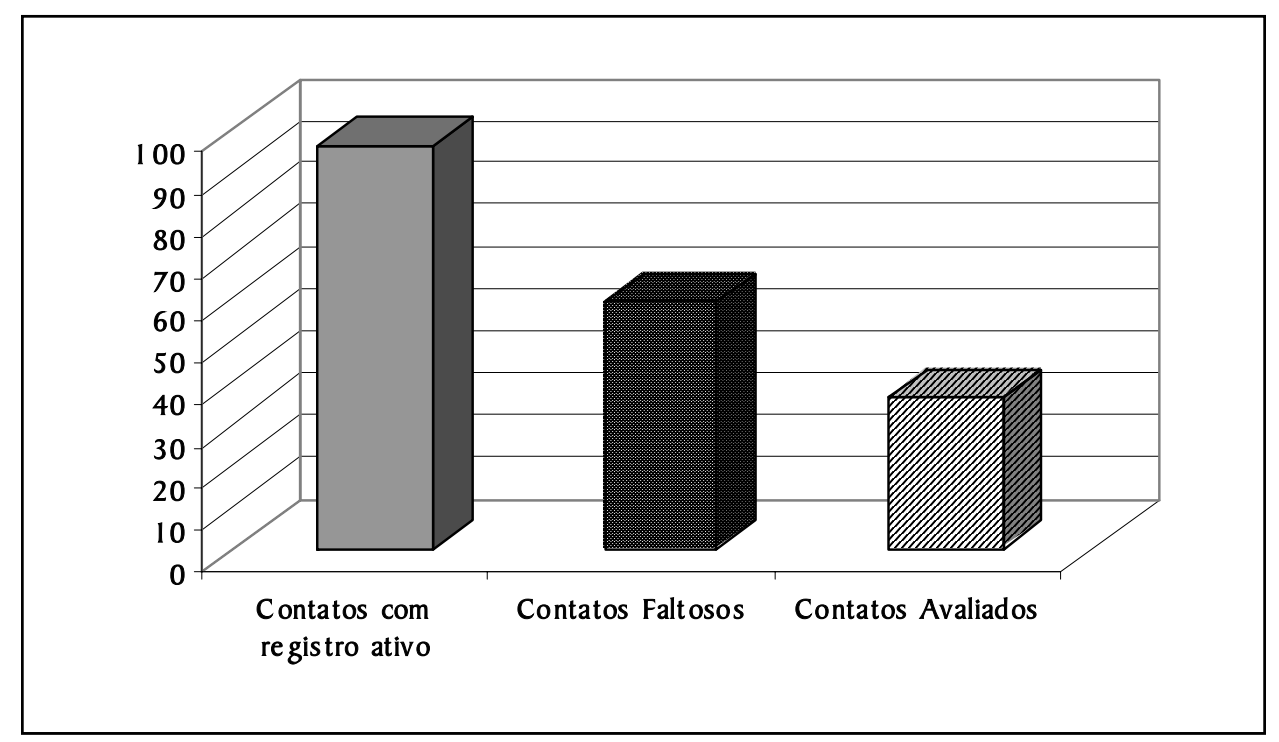

Figura 1. Distribuição dos contatos intradomiciliares de hanseníase, segundo o registro ativo, o percentual de faltosos e de avaliados em consulta de enfermagem Fonte: Ambulatório Regional de Especialidades de Taubaté de janeiro de 2003 a julho de 2004. 
Tabela 2. Distribuição dos contatos intradomiciliares faltosos de hanseníase $(n=23)$, segundo os motivos da não realização da consulta de enfermagem.

\begin{tabular}{lcc}
\hline Motivos & N & \% \\
\hline Recusaram a avaliação & 3 & 13,0 \\
Mudou de município & 4 & 17,4 \\
Mudou de residência & 2 & 8,7 \\
Não foi encontrado na residência & 10 & 43,5 \\
Transferido tratamento para outro município & 1 & 4,3 \\
Faleceram & 3 & 13,0 \\
\hline Total & $\mathbf{2 3}$ & $\mathbf{1 0 0 , 0}$ \\
\hline
\end{tabular}

resultados obtidos no estudo revelam as dificuldades encontradas para o controle de comunicantes de hanseníase e a não valorização Quanto importância da avaliação de controle.

Os serviços de saúde, após a implantação da PQT, têm realizado um esforço concentrado para diminuir a prevalência da hanseníase. No entanto, alguns problemas de ordem técnico operacional, ainda se interpõem para o alcance da meta de reduzir a prevalência por menos de um caso / 10.000 habitantes, destacando dentre eles, as baixas taxas de controle dos comunicantes ${ }^{(19)}$.

Outro dado significativo da não avaliação dos contatos foi Que 17,4\% deles mudaram de município, sendo este dado identificado somente no momento da visita domiciliária, para a realização da consulta de enfermagem, o Que demonstra a necessidade da unidade de saúde reforçar as ações de vigilância epidemiológica, valorizando a busca ativa, dado este também verificado em pesquisa realizada em São Luiz do Maranhão, no período de 1991 a 1995, onde apesar da hiperendemicidade do município, o programa vinha atuando de forma precária, necessitando de uma atenção maior no acompanhamento dos casos de hanseníase diagnosticados e registrados, e no exame dos contatos intradomiciliares ${ }^{(20)}$. Também foi significativo para o estudo, o número de contatos Que recusaram a avaliação (13\%), o Que nos leva a inferir sobre o seu desconhecimento Quanto a importância do controle.

A Figura a seguir, refere os motivos da não assiduidade à consulta de controle de contatos intradomiciliares, no Programa de Hanseníase.

A Figura 2 demonstra Que 66,7\%, não compareceu ao ARE para avaliação por motivo de esQuecimento; 11 , $1 \%$ por falta de tempo, $8,3 \%$ referiram ser por vergonha de comparecer ao serviço.

O estigma contra os pacientes de lepra afeta todos os aspectos de controle da doença; é preciso, portanto, conscientizar a população para tomar atitude com relação à hanseníase sem exagerar e nem minimizar as conseQüências da doença ${ }^{(21,22)}$. Existem vários fatores Que podem contribuir para a deficiência do controle das ações de hanseníase, e Que são ressaltados por estudioso da área Quando expressa: “...O diagnóstico tardio, a baixa cobertura assistencial, o abandono dos pacientes ao tratamento, baixa taxa de controle de comunicantes, baixo nível de esclarecimento da população. Também o estigma e o preconceito são fatores importantes ao penalizarem os portadores da doença, desta maneira, contribuem para dificultar a execução das medidas de controle" (19).

Reportando à pesquisa, pode-se deduzir Que o motivo das dificuldades do contato de hanseníase comparecer ao controle pode residir no fato das ações propostas para o seu acompanhamento serem preconizadas pelos órgãos públicos e definidas pelos profissionais de saúde como algo impositivo, para fazer cumprir, não considerando Que o contato também deveria participar de uma forma dinâmica da definição deste controle. Conforme parecer de estudiosos, membros do Ministério da Saúde, o estigma social associado à hanseníase contribuiu para o agravamento do problema no país ${ }^{(16)}$; fato este, Que confirma a negação de alguns contatos comparecerem ao ARE para avaliação, em função de conviverem com focos portadores de incapacidades físicas.

A enfermagem tem tido um papel fundamental Quanto ao controle da hanseníase, por meio da consulta de enfermagem, interagindo com o paciente/cliente, proporcionando a conscientização do controle de comunicantes intradomiciliares por meio da educação em saúde, e sobre a importância da vacinação do BCG-ID para prevenção e proteção para as formas mais contagiosas da doença. $\mathrm{O}$ Ministério da Saúde recomenda que os contatos intradomiciliares avaliados clinicamente, Que não tiverem contraído a doença, deverão ser encaminhados para receber a vacinação de BCG-ID. A vacina aumenta a resistência do organismo, principalmente contra as formas multibacilares da doença. Recomenda-se a aplicação de duas doses (tanto para os contatos de doentes da forma paucibacilar Quanto da multibacilar). A aplicação da segunda dose deve ser feita a partir de 6 meses da aplicação da primeira. Se o cliente já tiver cicatriz por vacina BCG-ID, esta deve ser considerada como a primeira dose, independente da época Que foi aplicada ${ }^{(2)}$.

Em relação à cobertura da vacina BCG ID encontrou-se, nas visitas realizadas, Que $66,6 \%$ dos contatos intradomiciliares de foco paucibacilar tinham as duas doses da vacina; e apenas 33,4\% tinham uma dose, não existindo, portanto nenhum caso destes contatos sem a vacina BCG-ID. Quanto ao foco multibacilar, identificou-se Que 54,2\% tinham as duas doses de BCG-ID; 41,6\% apenas uma dose e 4,2\% não possuíam nenhuma dose.

Fazendo-se uma correlação Quanto à assiduidade da aplicação do BCG-ID entre os contatos de paucibacilares e multibacilares, verificase um maior percentual de cobertura da vacina entre os paucibacilares, o Que pode ser explicado pelo período menor de controle destes contatos Que o dos multibacilares, ou seja, 2 anos, resultando em maior assiduidade ao serviço de saúde. Outra provável explicação para a não aderência ao controle dos contatos de multibacilares, e em conseQüência menor percentual de aplicação de BCG-ID neste grupo, deve-se ao fato do caso índice ter alta medicamentosa após um ano de tratamento, sendo Que seus contatos devem permanecer em avaliação de controle durante 5 anos.

Um outro fato que dificultou a coleta desses dados, na pesquisa, foi a descentralização da aplicação da vacina BCG-ID, do ARE Taubaté, com o encaminhamento dos contatos para imunização no Posto de Saúde próximo de sua residência, dificultando assim o acesso da equipe do Programa de Controle da Hanseníase à informação sobre a vacina, ocasionando falhas no registro da aplicação da BCG-ID, Que nem sempre expressavam a real situação deste controle.

Em relação à consulta de enfermagem, dos 36 contatos faltosos avaliados no domicilio, 4 foram encaminhados para avaliação médica, por apresentarem sinais suspeitos de hanseníase, sendo Que, I (um) teve comprovado o diagnóstico da doença, na forma Dimorfa (multibacilar). Este fato demonstra Que apesar de não se ter atingido 


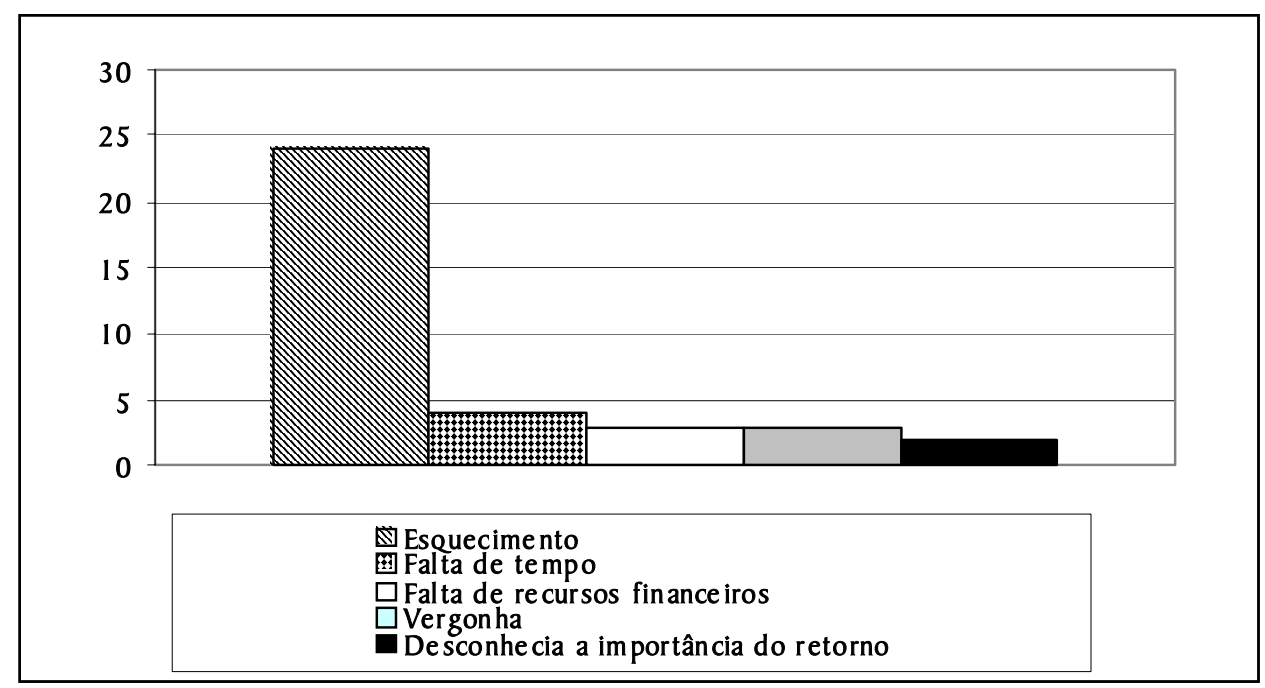

Figura 2. Distribuição dos contatos intra-domiciliares faltosos, segundo motivo do não comparecimento às consultas de controle Fonte: Ambulatório Regional de Especialidades Taubaté de janeiro de 2003 a julho de 2004.

100\% das consultas de enfermagem previstas, no curto espaço de tempo para desenvolver a coleta de dados, o resultado das avaliações foi significativo, pois se identificou um caso novo de hanseníase, auxiliando-se assim na Questão da busca ativa, com o objetivo de controle dos contatos e descoberta de casos novos.

Os resultados encontrados na pesQuisa são confirmados por outros estudos $^{(13,23,24)}$ cujos pesquisadores relatam: "A investigação epidemiológica inclui o exame das pessoas Que convivem ou conviveram, no domicílio ou fora dele, com o doente de hanseníase, QualQuer Que seja sua forma clínica, com o intuito de descobrir sua fonte de infecção e de detectar outros casos oriundos da mesma fonte ou do caso índice". "Apesar de ter sido diagnosticado apenas um caso de hanseníase nos comunicantes, epidemiologicamente, esse trabalho foi importante pela iniciativa de tentar descobrir possíveis focos de transmissão da hanseníase. Pioneiro no estado de Alagoas serviu como fonte de educação em saúde, permitindo-se desmistificar alguns tabus, inclusive o de Que a hanseníase não tem cura". "Um doente multibacilar, virgem de tratamento ou não tratado de maneira adequada, embora infectando muitas pessoas, produz cerca de cinco novos doentes por ano". Com base neste ultimo discurso, pode-se inferir que, nesta pesquisa, ao identificar um caso novo na forma multibacilar e encaminhá-lo para tratamento, contribuiu-se para evitar Que 5 novas pessoas adoecessem de hanseníase.

\section{CONSIDERAÇÕES FINAIS}

Diante dos resultados encontrados no estudo, fazem-se necessárias as seguintes considerações:

- Para a melhoria da organização e controle dos contatos de hanseníase no ARE Taubaté, verifica-se a necessidade de intensificar a busca ativa, melhorar o registro dos dados, e o controle da aplicação do BCG-ID, por meio da descentralização das ações de controle da doença;
- Devem-se estabelecer parcerias com outros serviços de saúde do município, como as unidades de PSF, ou com outras unidades básicas de saúde, no sentido de resgatar os contatos faltosos, fato este Que não ocorreu na organização do serviço estudado, assim como parcerias com outras instituições de saúde do município, com a finalidade de diagnóstico e tratamento precoce da doença.

- Existe a necessidade de focalizar e agilizar o diagnóstico de hanseníase em menores de 15 anos, pois podem ser contatos de casos ainda não-assistidos e não-identificados pelo sistema de saúde, como ocorreu de forma significativa nessa pesquisa, com esta população. Vale ressaltar a importância de implementar a busca ativa nesta faixa etária, visando o diagnóstico precoce da doença.

- Deve-se promover a educação continuada e capacitação da equipe multiprofissional da atenção básica visando diagnostico e tratamento precoce; bem como a formação de profissionais da área da saúde tanto do nível técnico como do nível superior de ensino.

- Sugere-se promover e implementar ações educativas, utilizando a mídia, profissionais da saúde, da educação e representantes de associações de bairros, com o objetivo de orientar doente, família e comunidade em geral, sobre aspectos clínicos, epidemiológicos e terapêuticos; prevenção de incapacidades, redução do estigma e preconceito.

- Faz-se necessária à continuidade deste trabalho de controle de contatos, em parcerias com as Universidades, sensibilizando e capacitando os acadêmicos da área de saúde, para suspeição diagnóstica e encaminhamento de casos suspeitos, visando identificar novos focos da doença, visto Que estes serão os futuros profissionais Que poderão colaborar com a eliminação da doença, uma vez Que a mesma tem um caráter crônico, com um longo período de incubação. Também se faz necessária à parceria com outros órgãos governamentais em nível federal, estadual e municipal, ONGs ou Instituições privadas, visando garantia de recursos financeiros e materiais para a efetivação do estudo. 


\section{REFERÊNCIAS}

1. Secretaria Municipal da Saúde (São Paulo). Doenças e agravos. [citado em: 2006 Nov 16]. Disponível em: http:// www2.prefeitura.sp.gov.br/secretarias/saude/vigilancia saude/ doenca agravo/000 I

2. Ministério da Saúde (BR). Secretaria de Políticas de Saúde. Departamento de Atenção Básica. Guia para o controle de hanseníase. Brasília: Ministério da Saúde; 2002.

3. Ministério da Saúde (BR). Secretaria de Políticas de Saúde. Departamento de Atenção Básica. Área Técnica de Dermatologia Sanitária. Hanseníase: atividade e controle. Brasília;: Ministério da Saúde; 2001

4. Ministério da Saúde (BR). Secretaria de Políticas de Saúde. Departamento de Atenção Básica. Controle de hanseníase na atenção básica. Brasília: Ministério da Saúde; 2001 .

5. Ministério da Saúde (BR). Secretaria de Políticas de Saúde. Departamento de Atenção Básica. Área Técnica de Dermatologia Sanitária. Manual de prevenção de incapacidades. Brasília: Ministério da Saúde; 2001.

6. Ministério da Saúde (BR). Área Técnica de Dermatologia Sanitária. Saúde para a vida: treinamento para prevenção de incapacidades em hanseníase: catalogador. Brasília: Ministério da Saúde; 1999.

7. Carrasco MAP, Pedrazzani ES. Situação epidemiológica da hanseníase e dos seus comunicantes em Campinas. Rev Esc Enferm USP 1993; 27(2): 124-8.

8. Instituto Brasileiro de Geografia e Estatística. Pesquisa mensal de emprego. Rio de Janeiro: IBGE; 2007.

9. Instituto Brasileiro de Geografia e Estatística. Resultados da amostra do censo demográfico 2000: malha digital do Brasil: situação em 200 I. Rio de Janeiro: IBGE; 2004.

10. Secretaria de Estado da Saúde. São Paulo. Decreto n. 43.379 de 13 de agosto de 1998. Dispõe sobre a reorganização do Centro de Saúde I de Taubaté Dr. Renée Rachou e dá providências correlatas. Diário Oficial do Estado de São Paulo; 1998 ago I4; Seção I: I.

11. Conselho Nacional de Saúde. Resolução n. 196 de 10 de outubro de 1996. Diretrizes e normas regulamentadoras de pesquisas envolvendo seres humanos. Bioética. 1996;4(2): 1525 (Supl).

12. Vieira LMM, Matos HJ, Duppre N, Alvin MFS, Sarno EM, Struchiner Cl. Epidemiologia da hanseníase em coorte de contatos intradomiciliares no Rio de Janeiro (1987-1991). Cad
Saúde Pública 1999; I 5(3): 533-42.

13. Opromolla DVA. Noções de hanseníase. Bauru: Centro de Estudos Dr. Reynaldo Quagliato; 2000.

14. Talhari S, Neves RG. Dermatologia tropical: hanseníase. Manaus: Editora Tropical; 1997.

15. Waldman EA, Silva LJ, Monteiro CA. Trajetória das doenças infecciosas da eliminação da poliomielite à reintrodução da cólera. In: Monteiro CA, organizador. Velhos e novos males da saúde no Brasil: a evolução do país e suas doenças. São Paulo: Hucitec; 2000. p. 105-244.

16. Evangelista $\mathrm{CMN}$. Fatores sócio-econômicos e ambientais relacionados à hanseníase no Ceará [dissertação]. Fortaleza: Faculdade de Medicina da Universidade Federal do Ceará; 2004.

17. Munhoz S, Fontes JF, Meireles SMP. Avaliação do programa de controle da hanseníase em municípios mato-grossenses. Rev Saúde Públ 1997; 3 I (3): 282-7.

18. Opromolla DVA. Organizações não governamentais brasileiras e o MORHAN. Hansen Int 1995; 20(2): I-2.

19. Lana FCF, Rocha SM. Organização do trabalho em hanseníase com a introdução da poliQuimioterapia. In: Almeida MCT, Rocha SM, organizadores. O trabalho de enfermagem. São Paulo: Cortez; 1997. p. II3-50.

20. Aquino DMC, Santos IS, Costa JML. Avaliação do programa de controle de hanseníase em um município hiperendêmico do Estado do Maranhão, Brasil, 1991-1995. Cad Saúde Pública 2003; 19(1): 119-25.

21. Baison KA, Van Den Borne B. Dimensions and processo of stigmatization in leprosy. Lepr Rev 1998; 69: 34I-50.

22. Salazar AR, Passa MC, Haernandez CM, Rujane M. El estigma en la representacion social de la lepra. Cad Saúde Pública 1995; 1 I (4): 2-4.

23. Nascimento Júnior CF, Correia DS, Gomes Filho Neto M, Bastos RPC. Busca ativa de hanseníase nos comunicantes intradomiciliares dos pacientes do programa de controle da hanseníase do Hospital Universitário/Universidade Federal de Alagoas. In: $2^{\circ}$ Congresso Brasileiro de Extensão Universitária; 2004 Set 12-15; Belo Horizonte (MG), Brasil. Belo Horizonte (MG): UFMG; 2004. [citado 2007 Nov 8 ]. Disponível em: http://www.ufmg.br/congrext/Saude/Saude48.pdf

24. Margarido LC, Rivitti EA. Hanseníase. In: Veronesi R, Focacia R. Tratado de infectologia. São Paulo: Atheneu; 2006. p. 937-69. 